\title{
THE EFFECT OF ZINC OXIDE FILLER ON MECHANO-PHYSICAL AND ELECTRO- MECHANICAL PROPERTIES OF PVDF
}

\author{
Miroslav MRLIK, Martin CVEK, Pavel BAZANT, Ivo KURITKA \\ Centre of Polymer Systems, University Institute, Tomas Bata University in Zlin, Czech Republic, EU, \\ $\underline{m r l i k @ u t b . c z}$
}

https://doi.org/10.37904/nanocon.2019.8637

\begin{abstract}
This study deals with the effect of star-like zinc oxide ( $\mathrm{ZnO}$ ) filler addition into poly(vinylidene fluoride) (PVDF) matrix on its structural and physical properties with the consequences to mechanical energy harvesting performance. In this case, the microwave-assisted synthesis was optimized for the preparation of unique starlike shape of $\mathrm{ZnO}$ particles. Their crystallinity and star-like morphology/elemental composition were analyzed using XRD and SEM/EDX spectroscopy, respectively. The investigation of $\alpha$-crystalline phase transformation into $\beta$-crystalline phase of neat PVDF matrix, and upon introducing various $\mathrm{ZnO}$ concentrations was performed using FTIR spectroscopy. Finally, the mechanical energy harvesting capability measurements showed that the addition of star-like $\mathrm{ZnO}$ filler enhanced the $d_{33}$ electro-mechanical coupling coefficient more than two times when compared to neat PVDF matrix.
\end{abstract}

Keywords: Poly(vinylidene fluoride), zinc oxide, polymorphism, crystallites, piezo-activity

\section{INTRODUCTION}

Semicrystalline polymer poly(vinylidene fluoride) (PVDF) is a material with unique properties possessing excellent film-forming, mechanical and thermal stability, tunable structural and also electrical properties [1-4]. These properties facilitate the utilization of this material in various applications such as controllable actuation, sensing or energy harvesting devices [5-7]. PVDF has unique semicrystalline character and especially crystalline phase is of importance for the potential applications. Electrically-active $\beta$-phase is one of three crystalline phases mainly occurring in the PVDF. In the case of $\alpha$-phase, the polymer chains are in alternating directions resulting in paraelectric behavior, therefore this phase is considered as a non-polar one. However, dominant occurrence $\beta$ - and $\gamma$-phase and suppression of non-polar $\alpha$-phase lead to polar behavior of the material [8-10]. Generally, PVDF forms the $\alpha$-crystalline phase which is useless in a view of mentioned applications. There are various methods to enhance the $\beta$-phase content in PVDF material even for already prepared films or non-processed granules. In the case of PVDF films, the useful methods include film stretching [11,12] or poling the films by the application of high external electric field (several $\mathrm{kV}$ ) [13]. The combination of both methods provides an improved $\beta$-phase content in comparison to the individual single method [14]. Another approach for $\beta$-phase development is the fabrication of PVDF films via electrospinning [15], or their PVDF co-blending with other polymers such as poly(methyl methacrylate) [16] or the introducing of various fillers forming the PVDF composites. These fillers can be organics (graphite, graphene oxide, reduced graphene) [1,17,18], or inorganic $\left(\mathrm{BaTiO}_{3}\right)$ [16]. This study is focused on the preparation of microwave-assisted zinc oxide $(\mathrm{ZnO})$ particles with unique star-like structure, and their influence on $\beta$-phase development in PVDF/ZnO composite films. Fabricated films were characterized from the structural point of view, and $\beta$-phase content was correlated with the amount of $\mathrm{ZnO}$ star-like filler. The suitability of prepared composites for intended applications was investigated using dielectric and dynamical mechanical analysis. Finally, harvesting performance of mechanical energy was analyzed in order to confirm the enhancing effects of ZnO star-like filler. 


\section{EXPERIMENTAL}

\subsection{Materials}

Zinc acetate dihydrate $\mathrm{Zn}\left(\mathrm{CH}_{3} \mathrm{COO}\right)_{2} \cdot 2 \mathrm{H}_{2} \mathrm{O}$ (ZAD), aqueous ammonia (25-29\%wt; $\mathrm{NH}_{3}$ aq.) were purchased from PENTA (Czech Republic). Acetone ( $\geq 99.5 \%$ ), N,N-dimethylformamide (99.8\%) (DMF), poly(ethylene glycol) (PEG; molecular weight of $400 \mathrm{~g} \cdot \mathrm{mol}^{-1}$ ), poly(vinylidene fluoride) (PVDF; molecular weight of $275000 \mathrm{~g} \cdot \mathrm{mol}^{-1}$ ) were purchased from Sigma-Aldrich (USA). All chemicals were of analytical grade and used as received. Demineralized (DEMI) water (conductivity of $25 \mu \mathrm{S} \cdot \mathrm{m}^{-1}$ ) was used during all experiments.

\subsection{Synthesis of $\mathrm{ZnO}$ star-like particles}

Microwave (MW) open-vessel system MWG1K-10 (Radan, Czech Republic) operating at $2.45 \mathrm{GHz}$ was used for MW hydrothermal synthesis with an external cooler. In the typical procedure, the starting materials were dissolved in DEMI water as follows: solution A was prepared by dissolution of $10.8 \mathrm{~g} Z A D$ in $80 \mathrm{~mL}$ of water, solution $B$ was prepared by dilution of $14.2 \mathrm{~mL}$ of $\mathrm{NH}_{3}$ aq. in $40 \mathrm{~mL}$ of DEMI water, and solution $\mathrm{C}$ contained $5.142 \mathrm{~g}$ of PEG dissolved in $20 \mathrm{~mL}$ of DEMI water. Reaction mixture was prepared by mixing solutions $\mathrm{A}$ and $\mathrm{C}$, followed by the addition of solution $\mathrm{B}$, while the total amount of added DEMI water was kept at $140 \mathrm{~mL}$ for syntheses. The solutions were poured into $250 \mathrm{~mL}$ reaction bottle and immediately placed into MW oven cavity connected to an external condenser, and exposed to MW irradiation for 10 minutes. Reaction mixture was left to cool down naturally. Finally, prepared particles were collected by microfiltration and several times washed by DEMI water. The obtained powders were dried under vacuum overnight.

\subsection{Preparation of PVDF/ZnO composite films}

The neat PVDF and PVDF/ZnO composite films with various content of $\mathrm{ZnO}$ star-like particles were fabricated via spin-coating technique. First, corresponding amount of $\mathrm{ZnO}$ particles was dispersed in $2 \mathrm{~mL}$ of DMF. The mixture was mechanically stirred using a glass stick and then ultrasonicated for 5 minutes. Subsequently, $1.4 \mathrm{~g}$ of PVDF pellets was added into the $\mathrm{ZnO}$ star-like particle solution together with the corresponding amount of DMF in order to achieve the same concentration for all the solutions. The mixtures were stirred at $60{ }^{\circ} \mathrm{C}$ until the complete dissolution of PVDF was reached. Then, the solutions containing $0.5,1,3,5$ and 10 wt.\% of $\mathrm{ZnO}$ star-like particles in comparison to PVDF were spin-coated on squared-glass substrates of dimensions $5 \mathrm{~cm}$ and covered by aluminum foil. A spin-coating process was optimized at the velocity of $2500 \mathrm{rpm}$ and the acceleration of $500 \mathrm{rpm} \cdot \mathrm{s}^{-1}$. In all cases, $1 \mathrm{~mL}$ of each solution was dripped on the substrate and the spin-coating process was performed under $\mathrm{N}_{2}$ atmosphere.

\subsection{Characterization}

Crystalline phase of $\mathrm{ZnO}$ star-like powder was characterized using X-ray diffractometer X'Pert PRO X-ray (PANalytical, The Netherlands) with a Cu-KaX-ray source $(\lambda=1.5418 \AA)$ in the diffraction angle range $2 \theta$ from $5^{\circ}$ to $85^{\circ}$. Infrared spectroscopy of neat PVDF and PVDF/ZnO films was performed using FTIR 670 Nicolet (Thermo Scientific, USA) in ATR mode. The data was collected in the wavenumber range from 4000 to 500 $\mathrm{cm}^{-1}$ to investigate $\beta$-phase development. The morphology of $\mathrm{ZnO}$ particles was observed using scanning electron microscopy (SEM) performed on FEI Quanta 200 Environmental Scanning Electron Microscope (ESEM) with a resolution of $5 \mathrm{~nm}$ and a magnification up to X200K. The device was equipped with energydispersive X-ray spectroscopy (EDX) to provide the elemental analysis of ZnO star-like filler.

\subsection{Energy harvesting setup}

To identify the electromechanical coupling constants of PVDF samples, a series of dynamic experiments was conducted. In the applied setup, PVDF sample was sandwiched between a conductive seismic mass on top 
and a conductive foil layer below. The seismic mass and the foil layer formed the electrodes with wires connecting them to the shunt resistance box. Beneath the foil layer is an insulating layer that isolates the sample from the metal shaker platform, which oscillates harmonically in the vertical direction. An accelerometer was used to measure the motion of the shaker table. A lumped parameter model of a piezoelectric device in the 3-3 mode shunted by a load resistance can be obtained according to our previous work [19].

\section{RESULTS AND DISCUSSION}

The described MW-assisted synthesis approach resulted in the development of 3D star-like ZnO superstructures (Figure 1). As seen in Figure 1a, this unique shape was developed for the vast majority of $\mathrm{ZnO}$ particles and also the particle size distribution seems to be very narrow. This feature is also confirmed in Figure 1b, where the star-like particles are displayed under a higher magnification. As can be clearly observed, their size was approximately $2.5 \mu \mathrm{m}$ in the diameter. Therefore, it can be stated that the MW-assisted synthesis provided a well-developed uniform star-like shape with narrow particle size distribution, which is highly required for the potential applications. In order to confirm the purity of the particles and the presence of ZnO structures, the EDX and XRD analyzes were performed (Figure 2). As obvious from the EDX investigations (Figure 2a), the star-like particles consisted of the expectable elements, i.e. zinc and oxygen. The low-energy spectrum region was dominated by the $\mathrm{L} \alpha$ emission peak for zinc and $\mathrm{K} \alpha$ peak for oxygen. Moreover, the emission peaks of $\mathrm{K} \alpha$ and $\mathrm{K} \beta$ for zinc appeared at high-energy region as typical for this element. The EDX spectrum exhibited the absence of peaks related to other elements, which indicates high purity of fabricated $\mathrm{ZnO}$ filler.
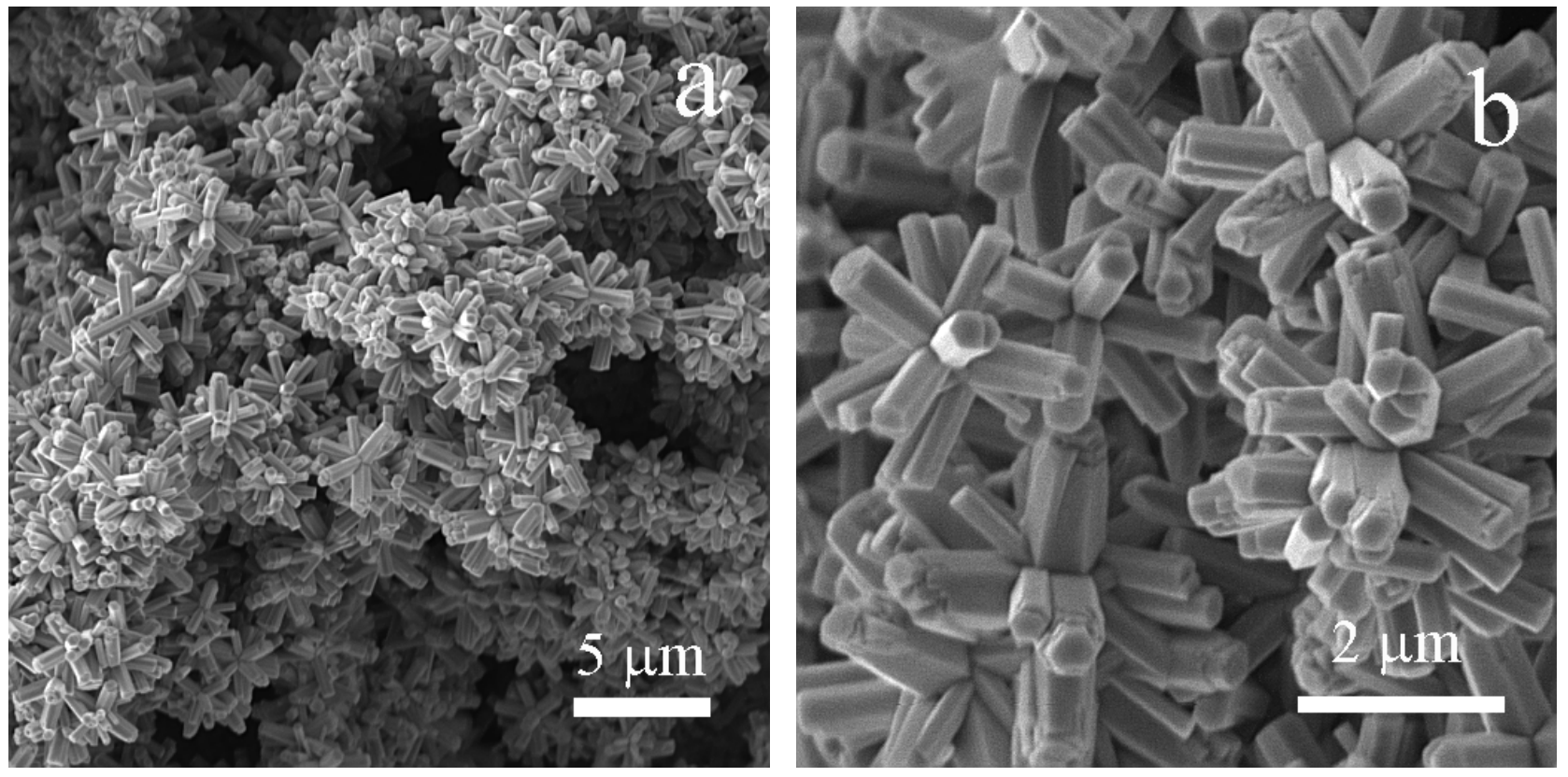

Figure $1 \mathrm{SEM}$ images of $\mathrm{ZnO}$ star-like particles under various magnifications

(a) 5000 and (b) 20000

The XRD investigations were performed to reveal the crystallography structure and further confirm high purity of $\mathrm{ZnO}$ star-like particles (Figure $\mathbf{2 b}$ ). Here, the diffraction peaks were centered at $2 \theta$ positions of $31.7^{\circ}, 34.4^{\circ}$, $36.2^{\circ}, 47.5^{\circ}, 56.6^{\circ}, 62.8^{\circ}, 67.8^{\circ}, 68.9^{\circ}, 72.47^{\circ}$ and $81.35^{\circ}$. Such combination perfectly matches with $\mathrm{ZnO}$ hexagonal wurtzite crystal structure according to JCDD PDF-2 entry 01-079-0207. This result was considered as a sufficient validation of high purity and $\mathrm{ZnO}$ crystalline structure of synthesized star-like particles. 

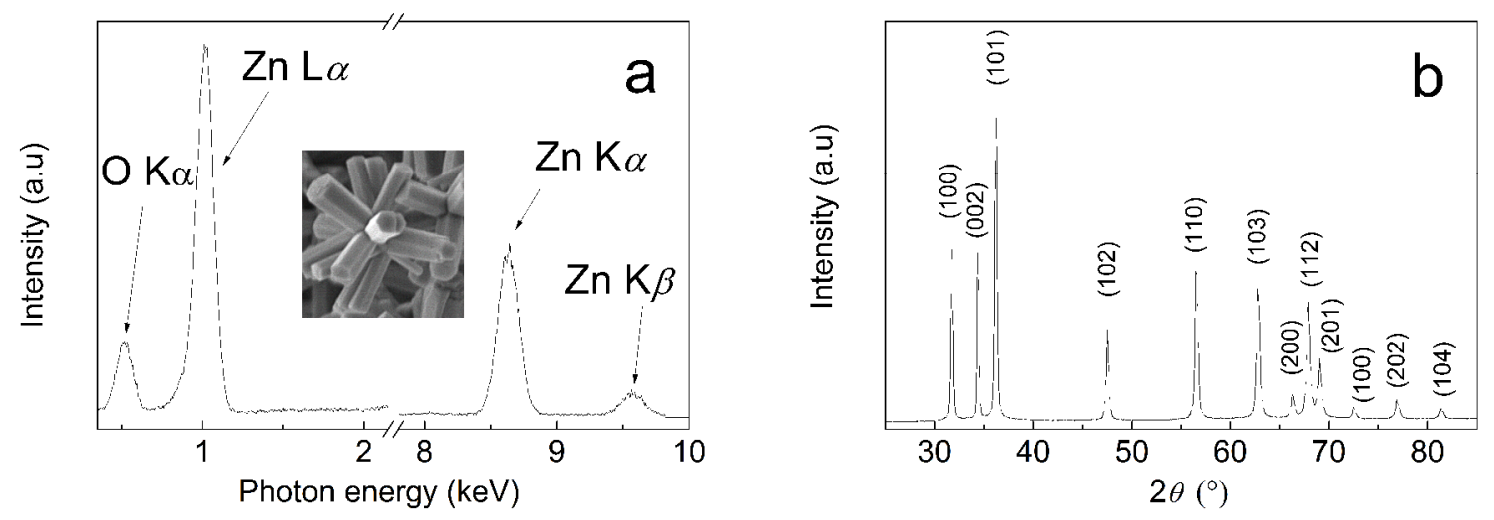

Figure 2 Energy dispersive spectrum (a) and X-ray diffraction spectrum (b) of ZnO star-like particles. Inset image shows the area from which the EDX spectrum was collected

The structural transformation of crystalline phases within PVDF and PVDF/ZnO composite films was investigated using FTIR spectra from $450 \mathrm{~cm}^{-1}$ up to $1500 \mathrm{~cm}^{-1}$ (Figure 3). In this case, the specific peaks for $\alpha$-phase are visible at 485, 611,762, 796, and $975 \mathrm{~cm}^{-1}$. The most dominating peaks for $\alpha$-phase are 611 and $762 \mathrm{~cm}^{-1}$, which represent bending and wagging vibration of $\mathrm{CF}_{2}$ group and rocking of the main PVDF chain, respectively. The absorption peaks at $836,874,1169,1232 \mathrm{~cm}^{-1}$ correspond to $\beta$-phase of PVDF. The sharp peak at $874 \mathrm{~cm}^{-1}$ represented the $\mathrm{CH}_{2}$ rocking and $\mathrm{CF}_{2}$ stretching, while the bands at 1171 and $1232 \mathrm{~cm}^{-1}$ reflected the wagging and rocking of $\mathrm{CH}_{2}$ group. The most important peaks regarding related to $\beta$-phase development and $\alpha$-phase suppression are positioned at $836 \mathrm{~cm}^{-1}$ and $762 \mathrm{~cm}^{-1}$, respectively. The relative ratios of peak intensity between these two absorption bands are summarized in Table 1 for neat PVDF film as well as for the composite films with various content of $\mathrm{ZnO}$ star-like particles. As can be seen, the peak ratio is 1.9 for neat PVDF, while with the increasing amount of $\mathrm{ZnO}$ star-like particles this ratio increasing and reaches the highest value of 22.5 for composite with $10 \mathrm{wt}$ \% filler content. This finding confirms the extensive development of $\beta$-crystalline phase as a consequence of $\mathrm{ZnO}$ incorporation.

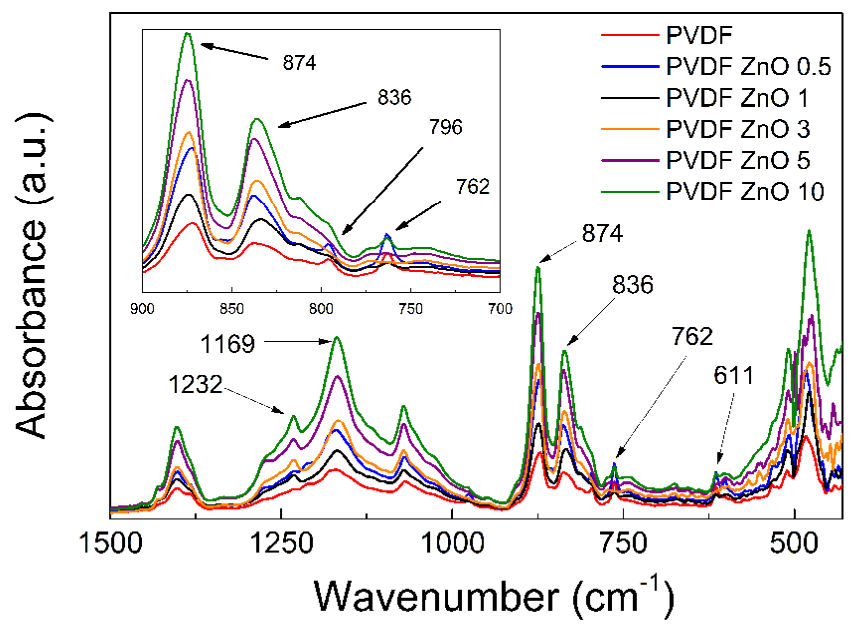

Figure $3 \mathrm{FTIR}$ spectra of neat PVDF and PVDF/ZnO composite films with various $\mathrm{ZnO}$ content

Table 1 Summarized values of relative ratio between the intensities corresponding to $\beta$ - and $\alpha$-phase

\begin{tabular}{|c|c|c|c|c|c|c|}
\hline Sample ID & Neat PVDF & $\begin{array}{c}\text { PVDF ZnO } \\
\mathbf{0 . 5}\end{array}$ & $\begin{array}{c}\text { PVDF Zno } \\
\mathbf{1}\end{array}$ & $\begin{array}{c}\text { PVDF Zno } \\
\mathbf{3}\end{array}$ & $\begin{array}{c}\text { PVDF Zno } \\
\mathbf{5}\end{array}$ & $\begin{array}{c}\text { PVDF ZnO } \\
\mathbf{1 0}\end{array}$ \\
\hline Ratio & 1.9 & 8.3 & 8.7 & 14.4 & 18.9 & 22.5 \\
\hline
\end{tabular}


The mechanical energy harvesting capability of materials based on PVDF mainly depends on the amount of $\beta$-phase developed in the individual samples [13]. As already mentioned, stretching or poling can be employed as possible strategies to induce $\beta$-phase crystallites and provide samples of suitable electro-active properties. In our case, neither of the mentioned methods was used for this purpose. Conversely, a facile spin-coating approach was utilized for composite film preparation. According to the amount of $\beta$-phase, the energy harvesting capability - represented by $d_{33}$ electro-mechanical coupling coefficient - noticeably increased with increasing amount of $\mathrm{ZnO}$ star-like particles (Table 2). The $d_{33}$ coupling coefficient increased more than two times from $2.35 \mathrm{pC} / \mathrm{N}$ to appreciable $5.18 \mathrm{pC} / \mathrm{N}$, for neat PVDF and PVDF ZnO 10, respectively, without any further fabrication steps.

Table 2 Summarized values of $d_{33}$ identification

\begin{tabular}{|c|c|c|c|c|}
\hline Sample ID & $\mathbf{1 0 k} \boldsymbol{\Omega}$ & $\mathbf{3 0 k} \boldsymbol{\Omega}$ & $\mathbf{5 0 k} \boldsymbol{\text { Average }}$ \\
\hline neat PVDF & $2.41 \mathrm{pC} / \mathrm{N}$ & $2.63 \mathrm{pC} / \mathrm{N}$ & $2.01 \mathrm{pC} / \mathrm{N}$ & $2.35 \mathrm{pC} / \mathrm{N}$ \\
\hline PVDF ZnO 1 & $2.69 \mathrm{pC} / \mathrm{N}$ & $3.00 \mathrm{pC} / \mathrm{N}$ & $3.03 \mathrm{pC} / \mathrm{N}$ & $2.91 \mathrm{pC} / \mathrm{N}$ \\
\hline PVDF ZnO 3 & $5.17 \mathrm{pC} / \mathrm{N}$ & $3.88 \mathrm{pC} / \mathrm{N}$ & $4.49 \mathrm{pC} / \mathrm{N}$ & $4.51 \mathrm{pC} / \mathrm{N}$ \\
\hline PVDF ZnO 5 & $5.22 \mathrm{pC} / \mathrm{N}$ & $4.71 \mathrm{pC} / \mathrm{N}$ & $4.46 \mathrm{pC} / \mathrm{N}$ & $4.80 \mathrm{pC} / \mathrm{N}$ \\
\hline PVDF ZnO 10 & $4.86 \mathrm{pC} / \mathrm{N}$ & $5.30 \mathrm{pC} / \mathrm{N}$ & $5.36 \mathrm{pC} / \mathrm{N}$ & $5.18 \mathrm{pC} / \mathrm{N}$ \\
\hline
\end{tabular}

\section{CONCLUSION}

In this study, the facile approach of polymer composite films preparation based on PVDF and ZnO star-like particles for mechanical energy harvesting was developed. Novel MW-assisted synthesis to fabricate the uniform $\mathrm{ZnO}$ star-like particles was introduced. These $\mathrm{ZnO}$ particles exhibited hexagonal wurtzite crystalline and high purity as confirmed via XRD and EDX, respectively. Thin PVDF/ZnO films with various $\mathrm{ZnO}$ content were prepared using spin-coating method, and the effect of $\mathrm{ZnO}$ content on structural transformation of crystalline phases was investigated. By quantifying FTIR spectra, it was found that the presence of ZnO starlike particles induced remarkable $\beta$-phase development. This structural transformation was reflected in $d_{33}$ electro-mechanical coefficient, which increased from $2.35 \mathrm{pC} / \mathrm{N}$ up to $5.18 \mathrm{pC} / \mathrm{N}$ after embedding $10 \mathrm{wt} . \%$ of $\mathrm{ZnO}$ particles. The presented concept of spin-coated PVDF/ZnO films appears to be highly promising to fabricate electro-active functional elements for low-voltage energy harvesting devices.

\section{ACKNOWLEDGEMENTS}

The authors gratefully acknowledge the Czech Science Foundation grant no. 19-17457S for the financial support. This work was also supported by the Ministry of Education, Youth and Sports of the Czech Republic - program NPU I (LO1504).

\section{REFERENCES}

[1] HUANG LY, LU CX, WANG F, WANG L, Preparation of PVDF/grahene ferroelectric composite films by in situ reduction with hydrobromic acids and their properties, RSC Advances, 2014, vol. 4, pp. 45220-45229.

[2] CHANG CE, TRAN VH, WANG JB, FUH YK, LIN LW, Direct-Write Piezoelectric Polymeric Nanogenerator with High Energy Conversion Efficiency, Nano Letters, 2010, 10, 726-731.

[3] ISSA, A. A. AI-MAADEED, M. A.S.A. MRLIK, M. and LUYT, A. S. Electrospun PVDF graphene oxide composite fibre mats with tuanble physical properties, Journal of Polymer Research, 2016, vol. 23, pp. 232 
[4] ISSA, A. A. AI-MAADEED, M. A.S.A. LUYT, A. S. MRLIK, M. and HASSAN, M. K. Investigatioin of the physicomechanical properties of electrospun PVDF/cellulose (nano) fibers, Journal of Applied Polymer Science, 2016, vol. 133, pp. 43594.

[5] LEE C, TARBUTTON JA, Electric poling-assisted additive manufacturing process for PVDF polymer-based piezoelectric device applications, Smart Materials and Structures, 2014, 23, 095044.

[6] SARAVANKUMAR B, SOVOON S, KIM SJ, Self-Powered pH Sensor Based on a Flexible Organic-Inorganic Hybrid Composite Nanogenerator, ACS Applied Materials and Interfaces, 2014, vol 6, pp. 13716-13723.

[7] FLORCZAK, S., LORSON, T., ZHENG, T., MRLIK, M., HUTMACHER, D.W. HIGGINS, M.J. LUXENHOFER, R., DALTON, P. D. Melt electrowriting of electroacitve poly(vinylidene fluoride) fibers, Polymer International, 2019, vol. 68, pp. 735-745.

[8] MARTINS P, LOPES AC, LANCEROS MENDEZ, S, Electroactive phases of poly(vinylidene fluoride): Determination, processing and applications, Progress in Polymer Science, 2014, 39, 683-706.

[9] MAJI S, SARKAR PK, AGGARWALI L, GHOSH SK, MANDALI D, SHEET G, ACHARYA S, Self-oriented betacrystalline phase in the polyvinylidene fluoride ferroelectric and piezo-sensitive ultrahin Langmuir-Schaefer film, Physical Chemistry Chemical Physics, 2015, 17, 8159-8165.

[10] KANIK, M, AKTAS O, SEN HS, DURGUN E, BAYINIR M, Spontaneous High Piezoelectricity in Poly(vinylidene fluoride) Nanoribbons Produces by Iterative Thermal Size Reduction Technique, ACS Nano, 2014, 8, 9311-9323.

[11] LI L, ZHANG MQ, RONG MZ, RUAN, WH, Studies on the transformation process of PVDF from alpha to beta phase by stretching, RSC Advances, 2014, 4, 3938-3943.

[12] LIU GM, SCHNEIDRER K, ZHENG LC, ZHANG XQ, LI CC, STAMM M, WANG DJ, Stretching induced phase separation in poly(vinylidene fluoride)/poly(butylene succinate) blends studies in-situ X-ray Scattering, Polymer, 2014, 55, 2588-2596.

[13] SHARMA M, MADRAS G, BOSE S, Process induced electroactive beta-polymorph in PVDF: effect on dielectric and ferroelectric properties, Physical Chemistry Chemical Physics, 2014, 16, 14792-14799.

[14] KIM GH, HONG SM, SEO Y, Piezoelectric properties of poly(vinylidene fluoride) and carbon nanotube blends: beta-phase development, Physical Chemistry Chemical Physics, 2009, 11, 10506-10512.

[15] FANG J, NIU HT, WANG HX, WANG XG, LIN, T, Enhanced mechanical energy harvesting sing needleless electrospun poly(vinylidene fluoride) nanofibre webs. Energy and Enviromental Science, 2013, vol. 6, pp. 21962202.

[16] MOKOFENG TG, LUYT AS, PAVLOVIC VP, PAVLOVIC VB, DUDIC D, VLAHOVIC B, DJOKOVIC V, Ferroelectric nanocomposites of polyvinylidene fluoride/ polymethyl methacrylate blend and $\mathrm{BaTiO} 3$ particles: Fabrication of beta-crystal polymorph rich matrix through mechanical activation of the filler. Journal of Applied Physics, 2014, vol. 115, pp. 084109.

[17] ZHANG YY, JIANG SL, YU Y, ZENG YK, ZHANG GZ, ZHANG QF, HE JG, Crystallization behavior and phasetranformation mechanism with the use of graphite nanosheets in poly(vinylidene fluoride) nanocomposites, Journal of Applied Polymer Science, 2012, vol. 125, pp. E314-E319.

[18] THANGAVEL E, RAMASUNDARAM S, PITCHAIMUTHU S, HONG SW, LEE SY, YOO SS, KIM DE, ITO E, KARIG YS, Structural and tribological characteristics of poly(vinylidene fluoride)/functionalized graphene oxide nanocomposite thin films, Composite Science and Technology, 2014, vol. 90, pp. 187-192.

[19] MRLIK, M., LEADENHAM, S., ALMAADEED, M-A. and ERTURK, A., Figure of merit comparison of PP-based electret and PVDF-based piezoelectric polymer energy harvesters, Proceedings of SPIE Active and Passive Smart Structures and Integrated Systems 2016, 2016, vol. 9799, pp- UNSP 979923. 